\title{
Batch Arrival Retrial Queue with Negative Customers, Multi-optional Service and Feedback
}

\author{
K. Kirupa \\ Research Scholar \\ Department of Mathematics, \\ Avinashilingam University, \\ Coimbatore, Tamil Nadu, India
}

\author{
K. Udaya Chandrika, PhD \\ Professor \\ Department of Mathematics, \\ Avinashilingam University, \\ Coimbatore, Tamil Nadu, India
}

\begin{abstract}
This paper deals with single server retrial queueing model where in addition to regular arriving customers there are negative arrivals. Server provides $M$ types of service. Positive customers arrive in batches according to Poisson process. If the server is idle upon the arrival of a batch, one of the customers in the batch receives any one of the types immediately and the rest join the orbit. Otherwise, the arriving batch joins the orbit. After completion of service, the customer may join the orbit as a feedback customer or depart the system. Negative arrival has the effect of removing the customer in service from the system. Performance analyses are obtained using supplementary variable technique. Stochastic decomposition law is verified and numerical results are also discussed.
\end{abstract}

\section{Keywords}

Retrial queue - negative customers -multi-optional servicefeedback

\section{INTRODUCTION}

The phenomenon of feedback in the retrial queueing system occurs in many practical situations. Many authors including Krishnakumar et al. [3], Mokaddis et al. [6] and Lee and Jang [4] analysed retrial queueing systems with feedback. Ramanath and Lakshmi [8] studied M/G/1 retrial queue with second multi-optional service and feedback. Recently, Baruah et al.[1] and Rajadurai et al.[7] analyzed a batch arrival retrial queueing system with two types of service, where server provides optional re-service.

Queues with negative arrivals called G-queues were first introduced by Gelenbe [2] with a view to modeling neural networks. In recent years, a variety of industrial applications have created interest in the modeling of reliability in G-queues. Liu et al.[5] analysed an M/G/1 retrial G-queue with server breakdown and feedback.

In this paper, we extend Baruah et al.[1] to retrial G-queue with multi-optional essential service and feedback.

\section{MODEL DESCRIPTION}

Consider a single server retrial queueing system with two types of independent arrivals, positive and negative. Positive customers arrive in batches according to Poisson process with rate $\lambda^{+}$. The batch size $\mathrm{Y}$ is a random variable with distribution function $\mathrm{P}(\mathrm{Y}=\mathrm{k})=\mathrm{C}_{\mathrm{k}}, \mathrm{k}=1,2, . ., \infty$ and probability generating function $\mathrm{C}(\mathrm{z})=\sum_{\mathrm{k}=1}^{\infty} \mathrm{c}_{\mathrm{k}} \mathrm{z}^{\mathrm{k}}$ having first two moments $\mathrm{m}_{1}$ and $\mathrm{m}_{2}$.
The server provides $M$ types of services. Customers opt the $\mathrm{i}^{\text {th }}$ type of service with probability $\mathrm{p}_{\mathrm{i}}(1 \leq \mathrm{i} \leq \mathrm{M})\left(\mathrm{p}_{1}+\mathrm{p}_{2}+\ldots+\mathrm{p}_{\mathrm{M}}=\right.$ $1)$. If the arriving batch of positive customers finds the server free, one of the arrivals receives service immediately. Otherwise the arriving batch joins the retrial queue. The retrial time is generally distributed with distribution function $\mathrm{A}(\mathrm{x})$, density function $\mathrm{a}(\mathrm{x})$, Laplace

Stieltje's transform $A^{*}(\theta)$ and conditional completion rate $\eta(x)$ $=\mathrm{a}(\mathrm{x}) /[1-\mathrm{A}(\mathrm{x})]$.

The service time of type $i(i=1,2, \ldots, M)$ follows a general distribution with distribution function $\mathrm{B}_{\mathrm{i}}(\mathrm{x})$, density function $b_{i}(x)$, Laplace Stieltje's transform $B_{i}{ }^{*}(\theta), n^{\text {th }}$ factorial moments $\mu_{i}^{(n)}$ and conditional completion rate $\mu_{i}(x)=b_{i}(x) /\left[1-B_{i}(x)\right]$, for $(\mathrm{i}=1,2, \ldots, \mathrm{M})$. After receiving service, the customer may join the orbit as a feedback with probability $\delta$ or depart the system with its complementary probability $1-\delta$.

Negative customers arrive singly according to Poisson process with rate $\lambda^{-}$. The arrival of a negative customer removes the positive customer being in service from the system and makes the server down. When the server fails, it stops providing service and is sent for repair immediately. The repair time also follows general distribution with distribution function $\mathrm{R}_{\mathrm{i}}(\mathrm{x})$, density function $r_{i}(x)$, Laplace Stieltje's transform $R_{i}^{*}(\theta), n^{\text {th }}$ factorial moments $\beta_{i}^{(n)}$ and conditional completion rate $\beta_{i}(x)=$ $r_{i}(x) /\left[1-R_{i}(x)\right]$, for $(i=1,2, \ldots, M)$. Various stochastic process involved in the system are independent of each other.

\section{ANALYSIS OF THE STEADY STATE DISTRBUTION}

The state of the system at time $t$ can be described by the Markov process $\{\mathrm{X}(\mathrm{t}), \mathrm{t} \geq 0\}=\{\mathrm{C}(\mathrm{t}), \mathrm{N}(\mathrm{t}), \xi(\mathrm{t}), \mathrm{t} \geq 0\}$ where $\mathrm{C}(\mathrm{t})$ denotes the server state $0, \mathrm{i}$ or $\mathrm{i}+\mathrm{M}$ according as the server being idle, busy or under repair. $\mathrm{N}(\mathrm{t})$ corresponds to the number of customers in the orbit. If $\mathrm{C}(\mathrm{t})=0$, then $\xi(\mathrm{t})$ represents the elapsed retrial time. If $\mathrm{C}(\mathrm{t})=\mathrm{i}$, then $\xi(\mathrm{t})$ represents the elapsed service time. If $C(t)=i+M$, then $\xi(t)$ represents the elapsed repair time of the failed server $(1 \leq \mathrm{i} \leq \mathrm{M})$.

\section{STEADY STATE DISTRBUTION}

For the process $\{C(t), t \geq 0\}$, define the probabilities

$$
\begin{aligned}
& I_{0}(t) \quad=P\{C(t)=0, N(t)=0\} \\
& I_{n}(x, t) d x=P\{C(t)=0, N(t)=n, x<\xi(t) \leq x+d x\}, n \geq 1 \\
& P_{n}^{(i)}(x, t) d x=P\{C(t)=i, N(t)=n, x<\xi(t) \leq x+d x\}, n \geq 0, \\
& i=1,2, \ldots, M
\end{aligned}
$$


$\mathrm{R}_{\mathrm{n}}^{(\mathrm{i})}(\mathrm{x}, \mathrm{t}) \mathrm{dx}=\mathrm{P}\{\mathrm{C}(\mathrm{t})=\mathrm{i}+\mathrm{M}, \mathrm{N}(\mathrm{t})=\mathrm{n}, \mathrm{x}<\xi(\mathrm{t}) \leq \mathrm{x}+\mathrm{dx}\}, \mathrm{n} \geq 0$, $\mathrm{i}=1,2, \ldots, \mathrm{M}$

The governing equations of the model under study in equilibrium state are

$\lambda^{+} \mathrm{I}_{0}=\sum_{\mathrm{i}=1}^{\mathrm{M}}\left[\bar{\delta}_{0}^{\infty} \int_{0}^{(\mathrm{i})}(\mathrm{x}) \mu_{\mathrm{i}}(\mathrm{x}) \mathrm{dx}+\int_{0}^{\infty} \mathrm{R}_{0}^{(\mathrm{i})}(\mathrm{x}) \beta_{\mathrm{i}}(\mathrm{x}) \mathrm{dx}\right]$

$\frac{\partial}{\partial x} I_{n}(x)=-\left(\lambda^{+}+\eta(x)\right) I_{n}(x), n \geq 1$

$\frac{\partial}{\partial \mathrm{x}} \mathrm{P}_{\mathrm{n}}^{(\mathrm{i})}(\mathrm{x})=-\left(\lambda+\mu_{\mathrm{i}}(\mathrm{x})\right) \mathrm{P}_{\mathrm{n}}^{(\mathrm{i})}(\mathrm{x})+\left(1-\delta_{0 \mathrm{n}}\right) \sum_{\mathrm{k}=1}^{\mathrm{n}} \lambda^{+} \mathrm{c}_{\mathrm{k}} \mathrm{P}_{\mathrm{n}-\mathrm{k}}^{(\mathrm{i})}(\mathrm{x})$,

$$
\mathrm{n} \geq 0, \mathrm{i}=1,2, \ldots, \mathrm{M}
$$

$\frac{\partial}{\partial x} R_{n}^{(i)}(x)=-\left(\lambda^{+}+\beta_{i}(x)\right) R_{n}^{(i)}(x)+\left(1-\delta_{0 n}\right) \sum_{k=1}^{n} \lambda^{+} c_{k} R_{n-k}^{(i)}(x)$,

$$
\mathrm{n} \geq 0, \mathrm{i}=1,2, \ldots, \mathrm{M}
$$

The boundary conditions are

$$
\begin{aligned}
& I_{n}(0)=\sum_{i=1}^{M}\left[\delta \int_{0}^{\infty} P_{n-1}^{(i)}(x) \mu_{i}(x) d x+\bar{\delta} \int_{0}^{\infty} P_{n}^{(i)}(x) \mu_{i}(x) d x\right] \\
& +\sum_{i=1}^{M} \int_{0}^{\infty} R_{n}^{(i)}(x) \beta_{i}(x) d x \\
& \mathrm{P}_{0}^{(\mathrm{i})}(0)=\mathrm{p}_{\mathrm{i}}\left[\lambda^{+} \mathrm{c}_{1} \mathrm{I}_{0}+\int_{0}^{\infty} \mathrm{I}_{1}(\mathrm{x}) \eta(\mathrm{x}) \mathrm{dx}\right], \mathrm{i}=1,2, \ldots, \mathrm{M} \\
& \mathrm{P}_{\mathrm{n}}^{(\mathrm{i})}(0)=\mathrm{p}_{\mathrm{i}}\left[\lambda^{+} \mathrm{c}_{\mathrm{n}+1} \mathrm{I}_{0}+\int_{0}^{\infty} \mathrm{I}_{\mathrm{n}+1}(\mathrm{x}) \eta(\mathrm{x}) \mathrm{dx}+\sum_{\mathrm{k}=1}^{\mathrm{n}} \lambda^{+} \mathrm{c}_{\mathrm{k}} \int_{0}^{\infty} \mathrm{I}_{\mathrm{n}-\mathrm{k}+1}(\mathrm{x}) \mathrm{dx}\right] \\
& \mathrm{R}_{\mathrm{n}}^{(\mathrm{i})}(0)=\lambda^{-}\left[\int_{0}^{\infty} \mathrm{P}_{\mathrm{n}}^{(\mathrm{i})}(\mathrm{x}) \mathrm{dx}\right], \mathrm{n} \geq 0, \mathrm{i}=1,2, \ldots, \mathrm{M}
\end{aligned}
$$

The normalizing condition is

$\mathrm{I}_{0}+\sum_{\mathrm{n}=1}^{\infty} \int_{0}^{\infty} \mathrm{I}_{\mathrm{n}}(\mathrm{x}) \mathrm{dx}+\sum_{\mathrm{n}=0}^{\infty} \sum_{\mathrm{i}=1}^{\mathrm{M}}\left[\int_{0}^{\infty} \mathrm{P}_{\mathrm{n}}^{(\mathrm{i})}(\mathrm{x}) \mathrm{dx}+\int_{0}^{\infty} \mathrm{R}_{\mathrm{n}}^{(\mathrm{i})}(\mathrm{x}) \mathrm{dx}\right]=1$

Define the probability generating functions

$\mathrm{I}(\mathrm{x}, \mathrm{z})=\sum_{\mathrm{n}=1}^{\infty} \mathrm{I}_{\mathrm{n}}(\mathrm{x}) \mathrm{z}^{\mathrm{n}}, \quad \mathrm{P}^{(\mathrm{i})}(\mathrm{x}, \mathrm{z})=\sum_{\mathrm{n}=0}^{\infty} \mathrm{P}_{\mathrm{n}}^{(\mathrm{i})}(\mathrm{x}) \mathrm{z}^{\mathrm{n}}$ and $R^{(i)}(x, z)=\sum_{n=0}^{\infty} R_{n}^{(i)}(x) z^{n}, i=1,2, \ldots, M$.

Multiplying equations (2) - (8) by $\mathrm{z}^{\mathrm{n}}$ and summing over all possible values of $n$, we obtain the following results:

$$
\begin{aligned}
& {\left[\frac{\partial}{\partial x}+\left(\lambda^{+}+\eta(x)\right)\right] I(x, z)=0} \\
& {\left[\frac{\partial}{\partial x}+\left(\lambda^{+}+\lambda^{-}-\lambda^{+} C(z)+\mu_{i}(x)\right)\right] P^{(i)}(x, z)=0} \\
& i=1,2, \ldots, M
\end{aligned}
$$

$\left[\frac{\partial}{\partial x}+\left(\lambda^{+}-\lambda^{+} C(z)+\beta_{i}(x)\right)\right] R^{(i)}(x, z)=0, i=1,2, \ldots, M$

$\mathrm{I}(0, \mathrm{z})=$
$\sum_{\mathrm{i}=1}^{\mathrm{M}}\left[\begin{array}{c}\mathrm{z} \delta \int_{0}^{\infty} \mathrm{P}^{(\mathrm{i})}(\mathrm{x}, \mathrm{z}) \mu_{\mathrm{i}}(\mathrm{x}) \mathrm{dx}+\bar{\delta} \int_{0}^{\infty} \mathrm{P}^{(\mathrm{i})}(\mathrm{x}, \mathrm{z}) \mu_{\mathrm{i}}(\mathrm{x}) \mathrm{dx} \\ +\int_{0}^{\infty} \mathrm{R}^{(\mathrm{i})}(\mathrm{x}, \mathrm{z}) \beta_{\mathrm{i}}(\mathrm{x}) \mathrm{dx}\end{array}\right]-\lambda^{+} \mathrm{I}_{0}(13)$ $\mathrm{P}^{(\mathrm{i})}(0, \mathrm{z})=\frac{\mathrm{p}_{\mathrm{i}}}{\mathrm{z}}\left[\int_{0}^{\infty} \mathrm{I}(\mathrm{x}, \mathrm{z}) \eta(\mathrm{x}) \mathrm{dx}+\lambda^{+} \mathrm{C}(\mathrm{z})\left[\begin{array}{l}\infty \\ \int_{0}^{\infty} \mathrm{I}(\mathrm{x}, \mathrm{z}) \mathrm{dx}+\mathrm{I}_{0}\end{array}\right]\right]$

$\mathrm{R}^{(\mathrm{i})}(0, \mathrm{z})=\lambda^{-} \int_{0}^{\infty} \mathrm{P}^{(\mathrm{i})}(\mathrm{x}, \mathrm{z}) \mathrm{dx}, \mathrm{i}=1,2, \ldots, \mathrm{M}$

Solving the partial differential equation (10) - (12), we get

$$
\begin{aligned}
& \mathrm{I}(\mathrm{x}, \mathrm{z})=\mathrm{I}(0, \mathrm{z}) \mathrm{e}^{-\lambda^{+} \mathrm{x}}[1-\mathrm{A}(\mathrm{x})] \\
& \mathrm{P}^{(\mathrm{i})}(\mathrm{x}, \mathrm{z})=\mathrm{P}^{(\mathrm{i})}(0, \mathrm{z}) \mathrm{e}^{-\left(\lambda^{+}+\lambda^{-}-\lambda^{+} \mathrm{C}(\mathrm{z})\right) \mathrm{x}}\left[1-\mathrm{B}_{\mathrm{i}}(\mathrm{x})\right], \\
& \mathrm{i}=1,2, \ldots, \mathrm{M} \\
& \mathrm{R}^{(\mathrm{i})}(\mathrm{x}, \mathrm{z})=\mathrm{R}^{(\mathrm{i})}(0, \mathrm{z}) \mathrm{e}^{-\left(\lambda^{+}-\lambda^{+} \mathrm{C}(\mathrm{z})\right) \mathrm{x}}\left[1-\mathrm{R}_{\mathrm{i}}(\mathrm{x})\right], \\
& \mathrm{i}=1,2, \ldots, \mathrm{M}
\end{aligned}
$$

Using equation (16) in equation (14), we get

$$
\mathrm{P}^{(\mathrm{i})}(0, \mathrm{z})=\frac{\mathrm{p}_{\mathrm{i}}}{\mathrm{z}}\left[\lambda^{+} \mathrm{I}_{0} \mathrm{C}(\mathrm{z})+\mathrm{I}(0, \mathrm{z})\left[\mathrm{C}(\mathrm{z})+(1-\mathrm{C}(\mathrm{z})) \mathrm{A}^{*}\left(\lambda^{+}\right)\right]\right],
$$

Using equation (17) in equation (15), we obtain $\mathrm{R}^{(\mathrm{i})}(0, \mathrm{z})=\lambda^{-} \mathrm{P}^{(\mathrm{i})}(0, \mathrm{z}) \overline{\mathrm{B}}_{\mathrm{i}} *(\mathrm{~g}(\mathrm{z}))$

where $\mathrm{g}(\mathrm{z})=\lambda^{+}+\lambda^{-}-\lambda^{+} \mathrm{C}(\mathrm{z})$

Using equations (17),(18),(19) and (20) in equation (13) and on solving we get

$$
\mathrm{I}(0, \mathrm{z})=\frac{\begin{array}{c}
\lambda^{+} \mathrm{I}_{0}\left[\mathrm { C } ( \mathrm { z } ) \sum _ { \mathrm { i } = 1 } ^ { \mathrm { M } } \mathrm { p } _ { \mathrm { i } } \left([\delta(\mathrm{z}-1)+1] \mathrm{B}_{\mathrm{i}}^{*}(\mathrm{~g}(\mathrm{z})) \mathrm{g}(\mathrm{z})\right.\right. \\
\left.\left.+\lambda^{-}\left(1-\mathrm{B}_{\mathrm{i}} *(\mathrm{~g}(\mathrm{z}))\right) \mathrm{R}_{\mathrm{i}} *(\mathrm{~h}(\mathrm{z}))\right)-\mathrm{zg}(\mathrm{z})\right]
\end{array}}{\mathrm{D}(\mathrm{z})}
$$

where,

$\mathrm{D}(\mathrm{z})=\mathrm{zg}(\mathrm{z})-\left(\left[\mathrm{A} *\left(\lambda^{+}\right)+\mathrm{C}(\mathrm{z})\left(1-\mathrm{A}^{*}\left(\lambda^{+}\right)\right)\right] \bullet\right.$

$$
\left.\sum_{\mathrm{i}=1}^{\mathrm{M}} \mathrm{p}_{\mathrm{i}}\left([\delta(\mathrm{z}-1)+1] \mathrm{B}_{\mathrm{i}}^{*}(\mathrm{~g}(\mathrm{z})) \mathrm{g}(\mathrm{z})+\lambda^{-}\left(1-\mathrm{B}_{\mathrm{i}} *(\mathrm{~g}(\mathrm{z}))\right) \mathrm{R}_{\mathrm{i}} *(\mathrm{~h}(\mathrm{z}))\right)\right)
$$
$\mathrm{h}(\mathrm{z})=\lambda^{+}-\lambda^{+} \mathrm{C}(\mathrm{z})$

Using equation (21), the equation (19) becomes

$\mathrm{P}^{(\mathrm{i})}(0, \mathrm{z})=\lambda^{+} \mathrm{I}_{0} \mathrm{~A}^{*}\left(\lambda^{+}\right) \mathrm{p}_{\mathrm{i}}[\mathrm{C}(\mathrm{z})-1] / \mathrm{D}(\mathrm{z})$

Using equation (22) in equation (20), we get

$\mathrm{R}^{(\mathrm{i})}(0, \mathrm{z})=$

$$
\lambda^{+} \lambda^{-} \mathrm{I}_{0} \mathrm{~A}^{*}\left(\lambda^{+}\right) \mathrm{p}_{\mathrm{i}}[\mathrm{C}(\mathrm{z})-1]\left[1-\mathrm{B}_{\mathrm{i}}^{*}(\mathrm{~g}(\mathrm{z}))\right] / \mathrm{D}(\mathrm{z})
$$

The partial probability generating function of the orbit size when the server is idle is given by

$$
\begin{aligned}
& \mathrm{I}(\mathrm{z})=\int_{0}^{\infty} \mathrm{I}(\mathrm{x}, \mathrm{z}) \mathrm{dx}= \\
& \left(\mathrm { I } _ { 0 } ( 1 - \mathrm { A } ^ { * } ( \lambda ^ { + } ) ) \left[\mathrm { C } ( \mathrm { z } ) \sum _ { \mathrm { i } = 1 } ^ { \mathrm { M } } \mathrm { p } _ { \mathrm { i } } \left([\delta(\mathrm{z}-1)+1] \mathrm{B}_{\mathrm{i}}^{*}(\mathrm{~g}(\mathrm{z})) \mathrm{g}(\mathrm{z})\right.\right.\right. \\
& \left.\left.\left.+\lambda^{-}\left(1-\mathrm{B}_{\mathrm{i}} *(\mathrm{~g}(\mathrm{z}))\right) \mathrm{R}_{\mathrm{i}} *(\mathrm{~h}(\mathrm{z}))\right)-\mathrm{zg}(\mathrm{z})\right]\right) / \mathrm{D}(\mathrm{z})
\end{aligned}
$$


The partial probability generating function of the orbit size when the server is busy in type i service is given by

$$
\begin{aligned}
& \mathrm{P}^{(\mathrm{i})}(\mathrm{z})=\int_{0}^{\infty} \mathrm{P}^{(\mathrm{i})}(\mathrm{x}, \mathrm{z}) \mathrm{dx}= \\
& \lambda^{+} \mathrm{I}_{0} \mathrm{~A}^{*}\left(\lambda^{+}\right) \mathrm{p}_{\mathrm{i}}(\mathrm{C}(\mathrm{z})-1)\left[1-\mathrm{B}_{\mathrm{i}}^{*}(\mathrm{~g}(\mathrm{z}))\right] / \mathrm{D}(\mathrm{z}), \mathrm{i}=1,2, \ldots, \mathrm{M}
\end{aligned}
$$

The partial probability generating function of the orbit size when the server is under repair is given by

$$
\begin{aligned}
& \mathrm{R}^{(\mathrm{i})}(\mathrm{z})=\sum_{\mathrm{i}=1}^{\mathrm{M}} \int_{0}^{\infty} \mathrm{R}^{(\mathrm{i})}(\mathrm{x}, \mathrm{z}) \mathrm{dx}= \\
& -\lambda^{-} \mathrm{I}_{0} \mathrm{~A}^{*}\left(\lambda^{+}\right) \mathrm{p}_{\mathrm{i}}\left[1-\mathrm{B}_{\mathrm{i}}^{*}(\mathrm{~g}(\mathrm{z}))\right]\left[1-\mathrm{R}_{\mathrm{i}}^{*}(\mathrm{~h}(\mathrm{z}))\right] / \mathrm{D}(\mathrm{z})
\end{aligned}
$$

The unknown constant $\mathrm{I}_{0}$ can be obtained by using the normalizing condition (9) as

$$
\mathrm{I}_{0}=\frac{\left.-\lambda^{-} \delta \sum_{\mathrm{i}=1}^{\mathrm{M}} \mathrm{p}_{\mathrm{i}} \mathrm{B}_{\mathrm{i}}^{*}\left(\lambda^{-}\right)-\lambda^{+} \lambda^{-} \mathrm{m}_{1} \sum_{\mathrm{i}=1}^{\mathrm{M}} \mathrm{p}_{\mathrm{i}} \beta_{\mathrm{i}}^{(1)}\right)\left(1-\mathrm{B}_{\mathrm{i}}^{*}\left(\lambda^{-}\right)\right)}{\lambda^{-} \mathrm{A}^{*}\left(\lambda^{+}\right)\left[1-\delta \sum_{\mathrm{i}=1}^{\mathrm{M}} \mathrm{p}_{\mathrm{i}} \mathrm{B}_{\mathrm{i}}^{*}\left(\lambda^{-}\right)\right]}
$$

Probability that the server is idle in the non-empty system is given by

$$
\begin{aligned}
& \left(1-\mathrm{A}^{*}\left(\lambda^{+}\right)\right)\left\{\lambda^{-}\left[\mathrm{m}_{1}-1+\delta \sum_{\mathrm{i}=1}^{\mathrm{M}} \mathrm{p}_{\mathrm{i}} \mathrm{B}_{\mathrm{i}}^{*}\left(\lambda^{-}\right)\right]\right. \\
& +\lambda^{+} \mathrm{m}_{1}\left[1+\sum_{i=1}^{\mathrm{M}} \mathrm{p}_{\mathrm{i}}\left[-\mathrm{B}_{\mathrm{i}}^{*}\left(\lambda^{-}\right)+\lambda^{-} \beta_{\mathrm{i}}^{(1)}\left(1-\mathrm{B}_{\mathrm{i}}^{*}\left(\lambda^{-}\right)\right)\right\}\right. \\
& \mathrm{I}=\lim _{\mathrm{z} \rightarrow 1} \mathrm{I}(\mathrm{z})=\frac{\mathrm{i}=1}{\lambda^{-} \mathrm{A}^{*}\left(\lambda^{+}\right)\left[1-\delta \sum_{\mathrm{i}=1}^{\mathrm{M}} \mathrm{p}_{\mathrm{i}} \mathrm{B}_{\mathrm{i}}^{*}\left(\lambda^{-}\right)\right]}
\end{aligned}
$$

Probability that the server is busy in type i service is given by

$$
\mathrm{P}=\lim _{\mathrm{z} \rightarrow 1_{\mathrm{i}=1}} \sum_{\mathrm{M}}^{\mathrm{M}} \mathrm{P}^{(\mathrm{i})}(\mathrm{z})=\frac{\sum_{\mathrm{i}=1}^{\mathrm{M}} \lambda^{+} \mathrm{m}_{1} \mathrm{p}_{\mathrm{i}}\left(1-\mathrm{B}_{\mathrm{i}}^{*}\left(\lambda^{-}\right)\right)}{\lambda^{-}\left[1-\delta \sum_{\mathrm{i}=1}^{\mathrm{M}} \mathrm{p}_{\mathrm{i}} \mathrm{B}_{\mathrm{i}}^{*}\left(\lambda^{-}\right)\right]}
$$

Probability that the server is under repair is given by

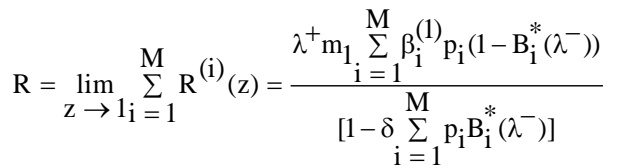

The probability generating function of the orbit size is

$\mathrm{P}_{\mathrm{q}}(\mathrm{z})=\mathrm{I}_{0} \mathrm{~A}^{*}\left(\lambda^{+}\right) \mathrm{g}(\mathrm{z})[\mathrm{z}-1]\left[1-\delta \sum_{\mathrm{i}=1}^{\mathrm{M}} \mathrm{p}_{\mathrm{i}} \mathrm{B}_{\mathrm{i}}^{*}\left(\lambda^{-}\right)\right] / \mathrm{D}(\mathrm{z})$

The probability generating function of the system size is

$\mathrm{P}_{\mathrm{s}}(\mathrm{z})=\mathrm{I}_{0} \mathrm{~A}^{*}\left(\lambda^{+}\right)[\mathrm{z}-1]\left[\lambda^{-}+(\mathrm{h}(\mathrm{z})-\delta \mathrm{g}(\mathrm{z})) \sum_{\mathrm{i}=1}^{\mathrm{M}} \mathrm{p}_{\mathrm{i}} \mathrm{B}_{\mathrm{i}}^{*}\left(\lambda^{-}\right)\right] / \mathrm{D}(\mathrm{z})$

\section{PERFORMANCE MEASURES}

Mean number of customers in the orbit is derived as

$\mathrm{L}_{\mathrm{q}}=\mathrm{P}_{\mathrm{q}}^{\prime}(1)=\frac{\mathrm{Nr}^{\prime \prime} \mathrm{Dr} \mathbf{r}^{\prime}-\mathrm{Nr}^{\prime} \mathrm{Dr} \mathbf{r}^{\prime \prime}}{2 \mathrm{Dr} \mathbf{r}^{2}}$

where $\operatorname{Nr}(\mathrm{z})$ and $\operatorname{Dr}(\mathrm{z})$ are the numerator and denominator of $\mathrm{P}_{\mathrm{q}}(\mathrm{z})$

$$
\mathrm{Nr}^{\prime}=\mathrm{I}_{0} \lambda^{-}\left[1-\delta \sum_{\mathrm{i}=1}^{\mathrm{M}} \mathrm{p}_{\mathrm{i}} \mathrm{B}_{\mathrm{i}}^{*}\left(\lambda^{-}\right)\right]
$$

$$
\begin{aligned}
\mathrm{Nr}^{\prime \prime}= & -2 \mathrm{I}_{0} \lambda^{+} \mathrm{m}_{1}\left[1+\lambda^{-} \delta \sum_{\mathrm{i}=1}^{\mathrm{M}} \mathrm{p}_{\mathrm{i}} \mu_{\mathrm{i}}^{(1)}-\delta \sum_{\mathrm{i}=1}^{\mathrm{M}} \mathrm{p}_{\mathrm{i}} \mathrm{B}_{\mathrm{i}}^{*}\left(\lambda^{-}\right)\right] \\
\operatorname{Dr}^{\prime}= & \lambda^{-}\left[1-\mathrm{m}_{1}\left(1-\mathrm{A}^{*}\left(\lambda^{+}\right)\right)-\lambda^{+} \mathrm{m}_{1}\left[1-\sum_{\mathrm{i}=1}^{\mathrm{M}} \mathrm{p}_{\mathrm{i}} \mathrm{B}_{\mathrm{i}}^{*}\left(\lambda^{-}\right)\right]\right. \\
& \left.-\lambda^{-} \delta \sum_{\mathrm{i}=1}^{\mathrm{M}} \mathrm{p}_{\mathrm{i}} \mathrm{B}_{\mathrm{i}}^{*}\left(\lambda^{-}\right)-\lambda^{+} \lambda^{-} \mathrm{m}_{1} \sum_{\mathrm{i}=1}^{\mathrm{M}} \mathrm{p}_{\mathrm{i}} \beta_{\mathrm{i}}^{(1)}\right)\left(1-\mathrm{B}_{\mathrm{i}}^{*}\left(\lambda^{-}\right)\right) \\
\operatorname{Dr}^{\prime \prime}= & -2 \lambda^{+} \mathrm{m}_{1}-\lambda^{+} \mathrm{m}_{2}-\lambda^{-} \mathrm{m}_{2}\left(1-\mathrm{A}^{*}\left(\lambda^{+}\right)\right) \\
& -\sum_{\mathrm{i}=1}^{\mathrm{M}} \mathrm{p}_{\mathrm{i}}\left[2 \lambda^{+} \lambda^{-} \mathrm{m}_{1} \delta \mu_{\mathrm{i}}^{(1)}-2 \lambda^{+} \mathrm{m}_{1} \delta \mathrm{B}_{\mathrm{i}}^{*}\left(\lambda^{-}\right)-2 \lambda^{+}{ }^{2} \lambda^{-} \mathrm{m}^{2} \mu_{\mathrm{i}}^{(1)} \beta_{\mathrm{i}}^{(1)}\right. \\
& +\lambda^{-}\left(1-\mathrm{B}_{\mathrm{i}}^{*}\left(\lambda^{-}\right)\right)\left[\lambda^{+} \mathrm{m}_{1}^{2} \beta_{\mathrm{i}}(2)+\lambda^{+} \mathrm{m}_{2} \beta_{\mathrm{i}}^{(1)}\right]-2 \lambda^{+} \mathrm{m}^{2} \mu_{\mathrm{i}}^{(1)} \\
& \left.-\lambda^{+} \mathrm{m}_{2} \mathrm{~B}_{\mathrm{i}}^{*}\left(\lambda^{-}\right)\right]-2 \mathrm{~m}_{1}\left(1-\mathrm{A}^{*}\left(\lambda^{+}\right)\right) \sum_{\mathrm{i}=1}^{\mathrm{M}} \mathrm{p}_{\mathrm{i}}\left[\left(\lambda^{-} \delta \mathrm{B}_{\mathrm{i}}^{*}\left(\lambda^{-}\right)\right.\right. \\
& \left.-\lambda^{+} \mathrm{m}_{1} \mathrm{~B}_{\mathrm{B}}^{*}\left(\lambda^{-}\right)+\lambda^{+} \lambda^{-} \mathrm{m}_{1} \beta_{\mathrm{i}}^{(1)}\left(1-\mathrm{B}_{\mathrm{i}}^{*}\left(\lambda^{-}\right)\right)\right]
\end{aligned}
$$

Mean number of customers in the system is

$\mathrm{L}_{\mathrm{s}}=\mathrm{P}_{\mathrm{s}}^{\prime}(1)=\frac{\mathrm{Nr}_{1}^{\prime \prime} \mathrm{Dr}^{\prime}-\mathrm{Nr}_{1}^{\prime} \mathrm{Dr}^{\prime \prime}}{2 \mathrm{Dr}^{\prime 2}}$

where $\mathrm{Nr}_{1}(\mathrm{z})$ and $\operatorname{Dr}(\mathrm{z})$ denotes the numerator and denominator of $\mathrm{P}_{\mathrm{s}}(\mathrm{z})$

$$
\begin{aligned}
& \mathrm{Nr}_{1}^{\prime}=\mathrm{I}_{0} \lambda^{-}\left[1-\delta \sum_{\mathrm{i}=1}^{\mathrm{M}} \mathrm{p}_{\mathrm{i}} \mathrm{B}_{\mathrm{i}}^{*}\left(\lambda^{-}\right)\right] \\
& \mathrm{Nr}_{1}^{\prime \prime}=-2 \mathrm{I}_{0} \lambda^{+} \mathrm{m}_{1}\left[\lambda^{-} \delta \sum_{\mathrm{i}=1}^{\mathrm{M}} \mathrm{p}_{\mathrm{i}} \mu_{\mathrm{i}}^{(1)}-(\delta-1) \sum_{\mathrm{i}=1}^{\mathrm{M}} \mathrm{p}_{\mathrm{i}} \mathrm{B}_{\mathrm{i}}^{*}\left(\lambda^{-}\right)\right]
\end{aligned}
$$

\section{RELIABILITY INDICES}

The system availability $\mathrm{A}(\mathrm{t})$ at time $\mathrm{t}$ is the probability that the server is either working for a customer or in an idle period. Then under steady state condition availability of the server is shown to be

$$
A=1-\sum_{\mathrm{i}=1}^{\mathrm{M}} \mathrm{R}^{(\mathrm{i})}=1-\frac{\lambda^{+} \mathrm{m}_{1} \sum_{\mathrm{i}=1}^{\mathrm{M}} \beta_{\mathrm{i}}^{(1)} \mathrm{p}_{\mathrm{i}}\left(1-\mathrm{B}_{\mathrm{i}}^{*}\left(\lambda^{-}\right)\right)}{\left[1-\delta \sum_{\mathrm{i}=1}^{\mathrm{M}} \mathrm{p}_{\mathrm{i}} \mathrm{B}_{\mathrm{i}}^{*}\left(\lambda^{-}\right)\right]}
$$

Steady state failure frequency of the server is

$$
F=\lambda^{-} \sum_{i=1}^{M} P^{(i)}=\frac{\lambda^{+} m_{1} \sum_{i=1}^{M} p_{i}\left(1-B_{i}^{*}\left(\lambda^{-}\right)\right)}{\left[1-\delta \sum_{i=1}^{M} p_{i} B_{i}^{*}\left(\lambda^{-}\right)\right]}
$$

\section{STOCHASTIC DECOMPOSITION}

Theorem: The decomposition property states that the number of customers in the system in steady state at a random point of time $\left(L_{s}\right)$ is distributed as the sum of two independent random variables, one of which is the number of customers in the corresponding standard queueing system in steady state at a random point of time $\mathrm{L}$ and the other random variable may have different probabilistic interpretation in specific cases depending on the vacation scheduled $\left(\mathrm{L}_{\mathrm{I}}\right)$.

Proof:

The probability generating function $\pi(\mathrm{z})$ of the system size in the classical batch arrival G-queue and unreliable server with feedback. Then equation (32) is given by 
$\pi(\mathrm{z})=$

$$
\begin{gathered}
{[\mathrm{z}-1]\left[\lambda^{-}+(\mathrm{h}(\mathrm{z})-\delta \mathrm{g}(\mathrm{z})) \sum_{\mathrm{i}=1}^{\mathrm{M}} \mathrm{p}_{\mathrm{i}} \mathrm{B}_{\mathrm{i}}^{*}\left(\lambda^{-}\right)\right]\left[\lambda^{-}-\lambda^{+} \mathrm{m}_{1}\left[1-\sum_{\mathrm{i}=1}^{\mathrm{M}} \mathrm{p}_{\mathrm{i}} \mathrm{B}_{\mathrm{i}}^{*}\left(\lambda^{-}\right)\right]\right.} \\
\frac{\left.\left.-\lambda^{-} \delta \sum_{\mathrm{i}=1}^{\mathrm{M}} \mathrm{p}_{\mathrm{i}} \mathrm{B}_{\mathrm{i}}^{*}\left(\lambda^{-}\right)-\lambda^{+} \lambda^{-} \mathrm{m}_{1} \sum_{\mathrm{i}=1}^{\mathrm{M}} \mathrm{p}_{\mathrm{i}} \beta_{\mathrm{i}}^{(1)}\right)\left(1-\mathrm{B}_{\mathrm{i}}^{*}\left(\lambda^{-}\right)\right)\right]}{\lambda^{-}\left[1-\delta \sum_{\mathrm{i}=1}^{\mathrm{M}} \mathrm{p}_{\mathrm{i}} \mathrm{B}_{\mathrm{i}}^{*}\left(\lambda^{-}\right)\right]\left[\mathrm{zg}(\mathrm{z})-\sum_{\mathrm{i}=1}^{\mathrm{M}} \mathrm{p}_{\mathrm{i}}\left([\delta(\mathrm{z}-1)+1] \mathrm{B}_{\mathrm{i}}^{*}(\mathrm{~g}(\mathrm{z})) \mathrm{g}(\mathrm{z})+\lambda^{-}\left(1-\mathrm{B}_{\mathrm{i}}^{*}(\mathrm{~g}(\mathrm{z}))\right) \mathrm{R}_{\mathrm{i}}^{*}(\mathrm{~h}(\mathrm{z}))\right)\right]}
\end{gathered}
$$

The probability generating function $\chi(\mathrm{z})$ of the number of customers in the orbit when the system is idle is given by

$$
\begin{aligned}
& \chi(\mathrm{z})=\frac{\mathrm{I}_{0}+\mathrm{I}(\mathrm{z})}{\mathrm{I}_{0}+\mathrm{I}(1)} \\
& {\left[\left[\mathrm{zg}^{\mathrm{z}} \mathrm{z}\right)-\sum_{\mathrm{i}=1}^{\mathrm{M}} \mathrm{p}_{\mathrm{i}}\left([\delta(\mathrm{z}-1)+1] \mathrm{B}_{\mathrm{i}}^{*}(\mathrm{~g}(\mathrm{z})) \mathrm{g}(\mathrm{z})+\lambda^{-}\left(1-\mathrm{B}_{\mathrm{i}} *(\mathrm{~g}(\mathrm{z}))\right) \mathrm{R}_{\mathrm{i}} *(\mathrm{~h}(\mathrm{z}))\right)\right] \bullet} \\
& {\left[\lambda^{-}\left[1-\mathrm{m}_{1}\left(1-\mathrm{A}^{*}\left(\lambda^{+}\right)\right)\right]-\lambda^{+} \mathrm{m}_{1}\left[1-\sum_{\mathrm{i}=1}^{\mathrm{M}} \mathrm{p}_{\mathrm{i}} \mathrm{B}_{\mathrm{i}}^{*}\left(\lambda^{-}\right)\right]-\lambda^{-} \delta \sum_{\mathrm{i}=1}^{\mathrm{M}} \mathrm{p}_{\mathrm{i}} \mathrm{B}_{\mathrm{i}}^{*}\left(\lambda^{-}\right)\right.} \\
& \left.-\lambda^{+} \lambda^{-} \mathrm{m}_{1} \sum_{\mathrm{i}=1}^{\mathrm{M}} \mathrm{p}_{\mathrm{i}} \beta_{\mathrm{i}}^{(1)}\right)\left(1-\mathrm{B}_{\mathrm{i}}^{*}\left(\lambda^{-}\right)\right)
\end{aligned}
$$

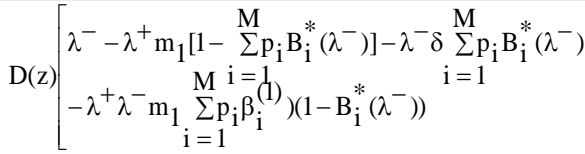

From equations (32),(37) and (38), it is observed that probability generating function of the number of customers in the system $\mathrm{P}_{\mathrm{s}}(\mathrm{z})$ is decomposed as $\mathrm{P}_{\mathrm{s}}(\mathrm{z})=\pi(\mathrm{z}) \chi(\mathrm{z})$.

$$
\text { Hence, } \mathrm{L}_{\mathrm{s}}=\mathrm{L}+\mathrm{L}_{\mathrm{I}} \text {. }
$$

\section{NUMERICAL RESULTS}

Numerical results are calculated by assuming the distributions of retrial times, service time and repair time as exponential with rates $\eta, \mu$ and $\beta$.

For the parameters $\lambda^{+}=0.2, \lambda^{-}=0.2, \mathrm{M}=3, \mathrm{p}_{1}=0.3, \mathrm{p}_{2}=0.3$, $\mathrm{p}_{3}=0.4, \mathrm{~m}_{1}=1.5, \mathrm{~m}_{2}=1, \delta=0.2, \eta=0.6, \mu_{1}=5, \mu_{2}=8, \mu_{3}=30, \beta_{1}=2$, $\beta_{2}=4, \beta_{3}=6$, the performance measures $\mathrm{I}_{0}, \mathrm{I}, \mathrm{P}, \mathrm{R}, A$ and $F$ are calculated and presented in tables 1 to 4 respectively for various rates of $\lambda^{+}, \lambda^{-}, \delta$ and $\eta$.

Table 1 reveals that $\mathrm{I}_{0}$ and $A$ monotonically decrease and I,P,R and $F$ increase as $\lambda^{+}$increases. Table 2 indicates that increase in $\lambda^{-}$increases $\mathrm{I}_{0}, \mathrm{R}$ and $F$ and decreases other performance measures. From Table 3 we observe that the parameters $\eta$ has no effect on the performance measures $\mathrm{P}, \mathrm{R}, A$ and $F$. As $\eta$ increases $\mathrm{I}_{0}$ increases and I decreases. Table 4 depicts the effect of $\delta$ on the performance measures. It is noted that I, P,R and $F$ increase and $\mathrm{I}_{0}$ and $A$ decrease for increasing values of $\delta$.

Figures 1 to 4 reveal the trend of Lq against the pair $\mathrm{i}-\left(\lambda^{+}\right.$, $\left.\mu_{1}\right)$, ii $-\left(\lambda^{-}, \mu_{2}\right)$, iii $-\left(\lambda^{-}, \beta_{1}\right)$ and iv $-\left(\delta, \mu_{1}\right)$.

Table. 1 Performance measures by varying $\lambda^{+}$

\begin{tabular}{|l|l|l|l|l|l|l|}
\hline $\boldsymbol{\lambda}^{+}$ & $\mathbf{I}_{\mathbf{0}}$ & $\mathbf{I}$ & $\mathbf{P}$ & $\mathbf{R}$ & $\boldsymbol{A}$ & $\boldsymbol{F}$ \\
\hline 0.1 & 0.8307 & 0.1478 & 0.0201 & 0.0015 & 0.9985 & 0.0040 \\
\hline 0.2 & 0.6542 & 0.3027 & 0.0401 & 0.0030 & 0.9970 & 0.0080 \\
\hline 0.3 & 0.4705 & 0.4648 & 0.0602 & 0.0045 & 0.9955 & 0.0120 \\
\hline 0.4 & 0.2797 & 0.6341 & 0.0802 & 0.0060 & 0.9940 & 0.0160 \\
\hline 0.5 & 0.0816 & 0.8106 & 0.1003 & 0.0075 & 0.9925 & 0.0201 \\
\hline
\end{tabular}

Table 2 Performance measures by varying $\lambda^{-}$

\begin{tabular}{|l|l|l|l|l|l|l|}
\hline $\boldsymbol{\lambda}^{-}$ & $\mathbf{I}_{\mathbf{0}}$ & $\mathbf{I}$ & $\mathbf{P}$ & $\mathbf{R}$ & $\boldsymbol{A}$ & $\boldsymbol{F}$ \\
\hline 0.3 & 0.6548 & 0.3013 & 0.0394 & 0.0044 & 0.9956 & 0.0118 \\
\hline 0.5 & 0.6560 & 0.2988 & 0.0381 & 0.0071 & 0.9929 & 0.0191 \\
\hline 0.7 & 0.6571 & 0.2964 & 0.0369 & 0.0096 & 0.9904 & 0.0258 \\
\hline 0.9 & 0.6582 & 0.2941 & 0.0358 & 0.0119 & 0.9881 & 0.0322 \\
\hline 0.1 & 0.6592 & 0.2920 & 0.0347 & 0.0141 & 0.9859 & 0.0382 \\
\hline
\end{tabular}

Table 3 Performance measures by varying $\eta$

\begin{tabular}{|c|l|l|l|l|l|l|}
\hline$\eta$ & $\mathbf{I}_{\mathbf{0}}$ & $\mathbf{I}$ & $\mathbf{P}$ & $\mathbf{R}$ & $\boldsymbol{A}$ & $\boldsymbol{F}$ \\
\hline 0.2 & 0.0488 & 0.9081 & 0.0401 & 0.0030 & 0.9970 & 0.0080 \\
\hline 0.4 & 0.5029 & 0.4540 & 0.0401 & 0.0030 & 0.9970 & 0.0080 \\
\hline 0.6 & 0.6542 & 0.3027 & 0.0401 & 0.0030 & 0.9970 & 0.0080 \\
\hline 0.8 & 0.7299 & 0.2270 & 0.0401 & 0.0030 & 0.9970 & 0.0080 \\
\hline 1.0 & 0.7753 & 0.1816 & 0.0401 & 0.0030 & 0.9970 & 0.0080 \\
\hline
\end{tabular}

Table 4 Performance measures by varying $\delta$

\begin{tabular}{|l|l|l|l|l|l|l|}
\hline$\delta$ & $\mathbf{I}_{\mathbf{0}}$ & $\mathbf{I}$ & $\mathbf{P}$ & $\mathbf{R}$ & $\boldsymbol{A}$ & $\boldsymbol{F}$ \\
\hline 0.1 & 0.7249 & 0.2337 & 0.0358 & 0.0027 & 0.9973 & 0.0072 \\
\hline 0.2 & 0.6542 & 0.3027 & 0.0401 & 0.0030 & 0.9970 & 0.0080 \\
\hline 0.3 & 0.5601 & 0.3908 & 0.0457 & 0.0034 & 0.9966 & 0.0091 \\
\hline 0.4 & 0.4358 & 0.5072 & 0.0530 & 0.0040 & 0.9960 & 0.0106 \\
\hline 0.5 & 0.2639 & 0.6682 & 0.0632 & 0.0047 & 0.9953 & 0.0126 \\
\hline
\end{tabular}

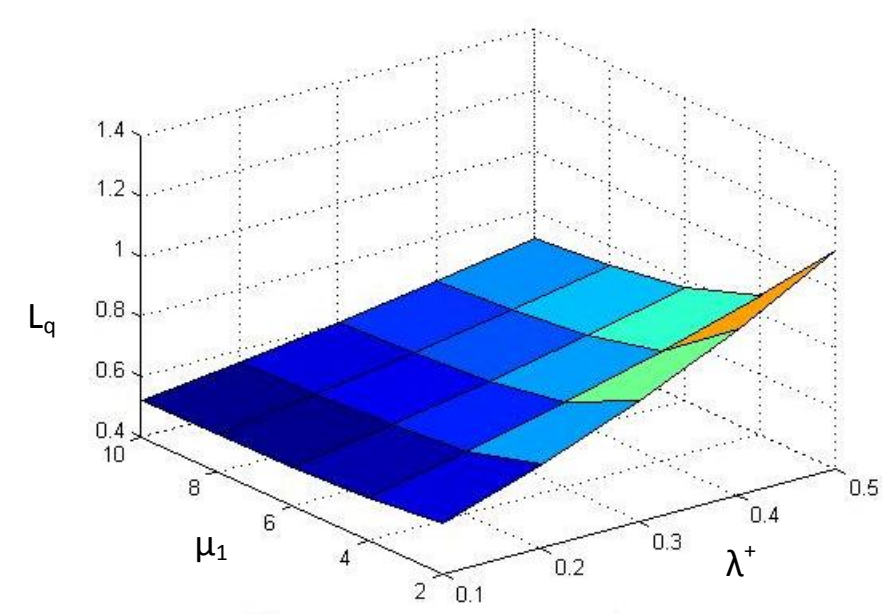

Figure 1- $L_{q}$ versus $\lambda^{+}$and $\mu_{1}$

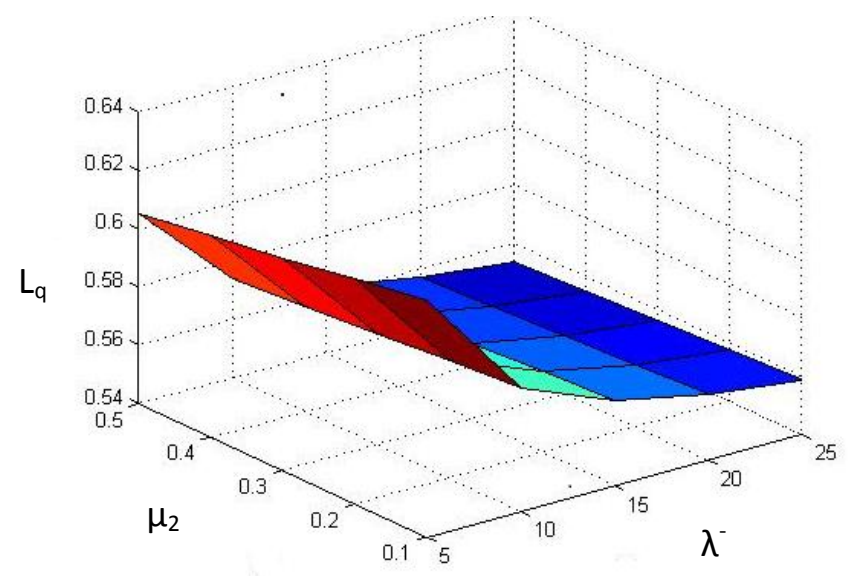

Figure 2- $L_{q}$ versus $\lambda^{-}$and $\mu_{2}$ 


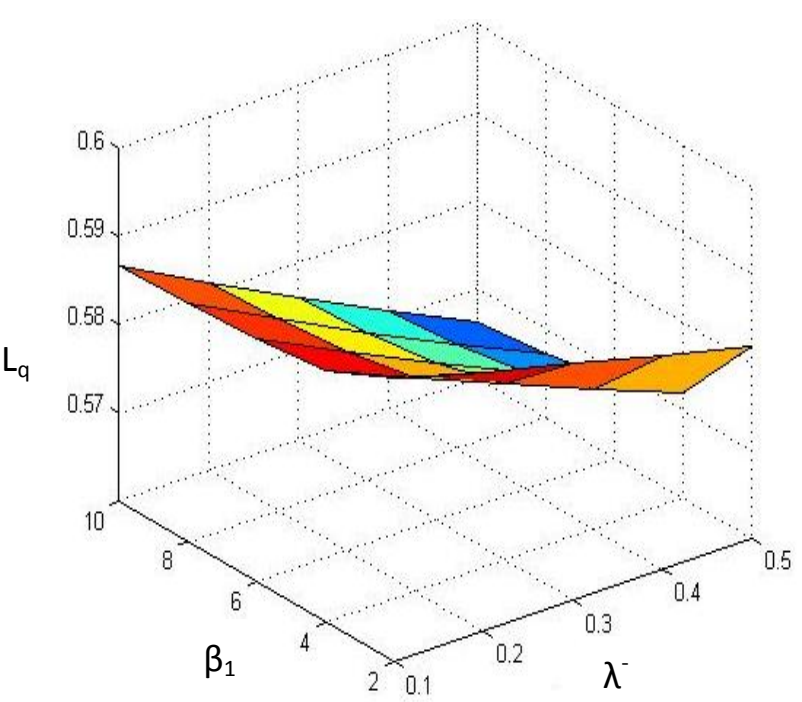

Figure 3- $L_{q}$ versus $\lambda^{-}$and $\beta_{1}$

\section{CONCLUSIONS}

In the present study, a single server batch arrival retrial queue with negative customers, multi-types of heterogenous service with feedback is analyzed. Analytical expressions for various performance measures are derived using supplementary variable technique. The stochastic decomposition law is verified. Numerical results are carried out to study the effect of some key parameters on the performance measures of the model. The vacation policy is analyzed in this paper is more general.

\section{REFERENCES}

[1] Baruah,M., Kailash C. Madan and Eldabi, T. 2012. On $\mathrm{M}^{[\mathrm{X}]} /\left(\mathrm{G}_{1}, \mathrm{G}_{2}\right) / 1$ queue with optional re-service, Applied Mathematical Computation.

[2] Gelenbe, E. 1989. Random Neural Networks with Negative and Positive Signals and Product Form Solution. Neural Computation 1, 502-510.

[3] Krishnakumar, B., Vijayalaksmi, G., Krishnamoorthy, A. and Sadiq Basha, S. 2010. A Single Server Feedback Retrial Queue with Collisions, Computers and Operations Research, Vol. 37, No. 7,1247 - 1255.

[4] Lee,Y.W. and Jang,Y.H. 2009. The M/G/ 1 Feedback Retrial Queue with Bernoulli Schedule,Journal of

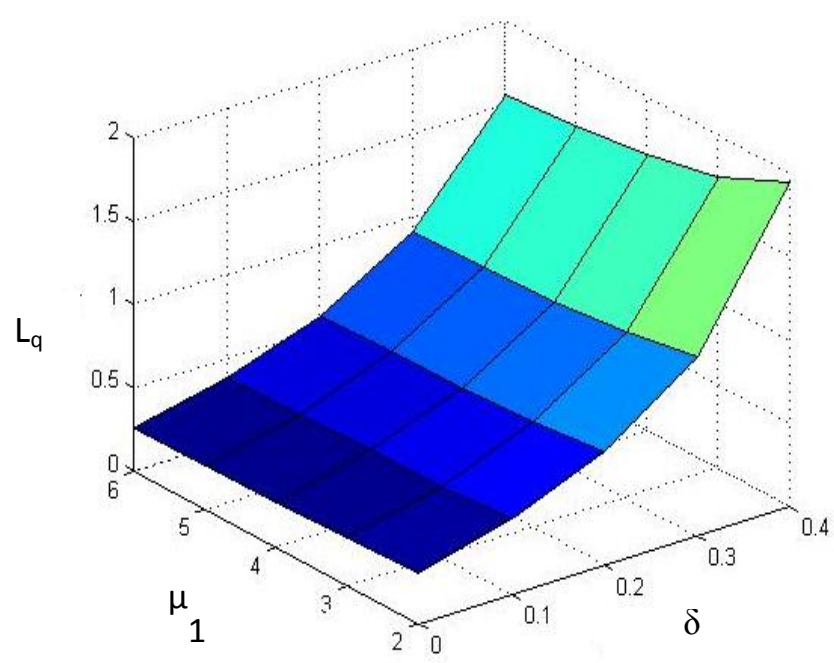

Figure 4- $\mathrm{L}_{\mathrm{q}}$ versus $\delta$ and $\mu_{1}$

Applied Mathematics and Informatics, Vol.27, No. 12, 259-266.

[5] Liu, Z., Wu, J. and Yang, G. .2009., An M/G/1 Retrial $\mathrm{G}$ - queue with Preemptive Resume And Feedback under N-policy Subject to Server Breakdowns and Repairs, Computers and Mathematics with Applications, 58, 1792-1807.

[6] Mokaddis, G.S., Metwally, S.A. and Zaki,B. M. 2007. A Feedback Retrial Queueing System with Starting Failures And Single Vacation, Tamkang Journal of Science and Engineering, Vol. 10, No.3, 183-192.

[7] Ramanath, K. and Lakshmi,K. (2011), An M / G / 1 Two Phase Multi - Optional Retrial Queue with Bernoulli Feedback and Non-Persistent Customers, International Journal of Latest Trends in Software Engineering, Vol.1, No.1,1-9

[8] Rajadurai, P. Saravanarajan, M. C. and Chandrasekaran, V. M. 2014. Analysis of an $\mathrm{M}^{[\mathrm{x}]} /\left(\mathrm{G}_{1}, \mathrm{G}_{2}\right) / 1$ Retrial queueing system with balking, optional re-service under modified vacation policy and service interruption, Ain Shams Engineering Journal, dx.doi.org/10.1016/j.asej.2014.02.00.3. 